\title{
Going Over normality-Health distinction: A New Overview
}

\section{Irtelli F* \\ Catholic university of the Sacred Heart, Milan, Italy}

*Corresponding author: Floriana Irtelli, Catholic university of the Sacred Heart, Largo Agostino Gemelli 1, Milano, Italy, Tel: 00393409272510; Email: dott.ssaflorianairtelli@gmail.com

\section{Perspective \\ Volume 3 Issue 7}

Received Date: August 09, 2018

Published Date: September 04, 2018

DOI: $10.23880 /$ pprij-16000180

\section{Abstract}

A lot of interdisciplinary discussions have been opened about the topic of the health and its definitions, this happened also because often the concepts of health and normality are used in interchangeable way. Not in line with a such conception, and encouraged by the demand to find alternative solutions, in this paper has been analyzed some epistemic assumptions and the distinction between these concepts; these terms have been first examined in their historical and conceptual evolution, focusing on how the notion of health in past was founded on some dichotomic points of view, and then analyzing how today has been privileged a new dynamic perspective. Unfortunately also the concept of normality is often still based on some old dichotomic distinctions, not useful today; the conclusions of this paper point out the difference and distinction of value among these terms and finally put on in evidence the importance to go over a dichotomic vision for the definitions of the health and normality, to conceive anew bivariate model, and finally explain how the concept of normality can be replaced by the new concept of normativity.

Keywords: Health; Normality; Normativity; Conceptual Analysis; Distinction; Bivariate Model; Dichotomy

\section{Epistemic Assumptions}

It is necessary to set some precise conceptual distinctions and to explain you determine terminologies to favor a clear scientific dialogue among different disciplines, since interdisciplinary research, as valuable as it certainly is, seems to be to fundamentally ambiguous endeavor. Such ambiguity, importantly, does not consist in the very idea of building bridges between disciplines. Rather, it refers to theoretical and methodological issues inevitably involved in connecting two or more field. The latter are often substantially different and may involve conceptual and theoretical apparatuses which, if not altogether incommensurable, are hard to translate into each other [1]. The terminologies used in the professional health care and in health science is partly unclear [2], and also distinguish normal from disturbed conditions, appears an extremely confused operation in mental health [3]. Normality is an element of sense making that changes and develops over time, and experiential information and social contexts as central to healthrelated sense making [4]. On the base of these recent and interesting observations has been opened some reflections regarding the interlacement and the comparison on the concepts of Normality and Health, also making reference to the historical evolution of the theories, and to that movement for the mental Health to whose base revealed him that that results "an ideological component" [5] that we can define normative. It is for example concretized in the project to recognize and to eradicate that aspects of personality that were the site where the seeds future of individual and social problems were sown and germinated [6] and, accordingly, 


\section{Psychology \& Psychological Research International Journal}

established as an object of intervention [1]. We consider that the personality, in its characteristics, can introduce considerable deviations from the statistic norm, without for this to have to believe that these deviations are not "healthy" [1]. As a matter of fact in different studies have been criticized the interchangeability and little clarity of the notions of normality and health, since the two notions are not identical. These concepts are often used in interchangeable way and the difference among them it is often vague: there is the necessity of an explanation about the specificities of these two notions. The interdisciplinary search seems to be fundamental with the purpose to make an effort to go further to conceptual ambiguity proper of the daily language.

For this reflection is important to refer to clear conceptual and theoretical approaches like the biopsychosocial model [7-9] and the psychosomatic one [10-11]: these seem useful references for the theme in object. Today the biopsychosocial model is the privileged paradigm to decode and to understand the health [7-9]. In the years when this paradigm was born there has been a passage from a "traditional biomedical model", heralding of a strong tendency to the reductionism (the dogmatic philosophical perspective, in base to which a complex phenomena derives from a single primary principle), to the paradigm of the complexity, on which the biopsychosocial perspective is founded. The old traditional biomedical model is well described from Engel [7]: this approach assumes that health can be fully explained as deviation by the statistic norm of measurable variables. The biomedical model requires that health is treated as independent from the social behavior, and it also pretends that behavioral deviations can be explained on the base of disturbed somatic trials. This model embraces both the reductionism and the dualism mindbody: doctrines that separate the mental aspects from the somatic aspects. However toady we know that this separation belongs more to a classificatory need that to a realistic point of view (that conceives body and mind as a whole). According to Lipowsky [10] also the concept of single causes and sequences is now superficial and obsolete [12]. Such perspective is proper of an outdated epistemology that gives just an explanation of the physical and psychic phenomena through the individualization of relationships of linear cause-effect, predictable and controllable. The biomedical paradigm is based on these inadequate principles, so its limits have been criticized among the years Sixty and Seventy of last century; as a matter of fact in this period a new epistemology was born, its name is the epistemology "of the complexity": such point of view implicates the overcoming of the old conception of mind and body as separate factors; instead, according to the new perspective the health emerges from an interaction of multiple factors with retroactive effects on each other [7-9]. This last perspective (which sees the psychological, social and biological factors intertwined) has recently been confirmed by a great body of scientific researches, for example the experimental results about the placebo effect: $35-40 \%$ of the positive answers to the treatment of the illnesses in the medical practice can be explained by such effects [13]. A lot of scientific achievements have allowed criticizing the concept of health expressed by the biomedical model and its deterministic theories [7]: the desire to subdue the complexity of the human becoming to the simple causality has brought therefore to contemplate a mechanistic conceptual frame, that reduces the multiplicity of the existence to few linear categories, few labels. The deterministic positions appear as invested by a search of determination and control that today are obsolete. Having clarified these assumptions now we can ask ourselves, like Gadamer: "what is the health, this mysterious condition that all know and that on the other hand we don't know at all?" [14]. for example Freud has expressed health as the ability to work and to love $[15,16]$ according to the theoretical vision of the beginning ' 900 , isolating and reducing the variables [7-17]. Today we can say that it is a problem to reduce the variables and their complexity. Founding ourselves on new approaches we can deepen the notions of normality and health that will be object of investigation, both historically and systematically. A parallel will be delineated among the couple of concepts to make a clear distinction and to underline the importance to go over a dichotomic vision for the definition of the health, and therefore the importance to conceive a bivariate model, both as the concept of normality can be placed side by side by the new concept of normativity.

\section{Historical Excursus about the Notion of Health}

These concepts assumed a great relief for the whole humanity, for a long time; they deal with the values, the habits, the styles of life of different social contexts, since the sense of the health and normality are correlated to the culture and the interaction among social, psychological and physiological aspects [18]. To be more aware of the actual representations of the health and normality we can analyze the past epochs points of view, starting from primitive conceptualizations with strong influences of religious experience. As we will see in ancient times, health has been conceived as a gift from God, or as a 


\section{Psychology \& Psychological Research International Journal}

balance between vital forces, or a complementary presence of instances. These ancient implicit conceptions that have constituted our specific way to think about the to these concepts through progressive transformations: the etymological dimension of the word "health" derives from the Latin Salus that means: fortune, prosperity, life, salvation, safety, and also in the Jewish tradition, the unique and transcendent God rewarded the men with the health. After the first moral connotations the health loses then the personal connotations, affirming itself substantially as equilibrium and harmony. In the Pythagorean school Alcmeone considered health like the equilibrium among organic strengths: damp and dry, heat and cold, bitterness and sweetness. Analogous it was the position of Hippocrates. The concept of equilibrium and harmony was also a particularly diffused conception in the Indian culture, in which earth, water, fire, wind and ether are the five elements that constitute the subject and guarantee the good health. In the Greek mythology, subsequently, the god Aesculapius had two daughters: one called Igea and the other Panacea. While this last represented the "care" for the illness, Igea was the goddess of the health. She taught to the Greek how to be "healthy." These figures symbolically express the presence of two complementary appeals, among which it would be artificial to attribute patent of superiority or subordination. The relationship between these two appeals can sometimes suffer cultural historical variations with unbalanced equilibriums in the one or in the other direction $[19,20]$. As anticipated, it is possible to focus on three different models of representation of the health: the first one centered on moral dimensions and endogenous factors in the genesis of the Health. The second conception is based on the relationships of equilibrium among the parts, and on the connections in a whole (the integrity of a living system). The third conception makes reference to the co presence among appeals, and it will be the precursory of the contemporary bivariate model [21-27]. The beliefs of the origins constitute anticipations of the following theories, and at the beginning of the new century have been delineated most suitable tools for the investigations and the observation of this concept.

\section{Historical excursus about the notion of normality}

The concept of normality can be defined by different points of view and has been conceptualized over time as being akin to an ideal, or belonging to a category, or has been defined on the basis of statistical parameters. "Normal" is often understood, or misunderstood, as "ideal", and Homer W. Smith, pointed out in a lecture at the university of the California (from the title "Plato and Clementine", published in the Bulletin in the New York Academy of Medicines, 1947) that Plato blames for this misunderstanding, for which the Normality could refer to an ideal Platonic essence, as the perfect beauty or the perfect body [28]; the normality can also mean adequacy, from the definition of Plato, in the sense to be without defects lacks or disorders: in an optimal state [28]. Another way to understand normality was to create value thresholds that discriminated against what is normal or not; this happened because the western thinkers have spent a considerable dose of energy to organize the understanding of the world in a series of "separate things", sectioning the reality in categories. As a matter of fact Wright and Lopez [29] emphasized that a product of categorizations is the attenuation of the differences ingroup (de-individualization) and the accentuation of the differences inter-group. An example of this point of view can be Ann Freud's theory, tracing a line of border of the normality, underlining the distinction between more primitive defenses and more evolved defenses (which are a sign of normality), showing her increasing interest in to build a systematic point of view on this theme, making a classification of what is normal and what is not: according to her theory, the excess of some a mechanism of defense is labeled as indicator of the lack of normality [30,31]. This term could mean, also "in the mathematical average", for example making reference to the middle weight or the height of a population: the notion of normality points out the most common aspect inside a population, for example to have the brown eyes in some countries or the blue eyes in the northern countries. The Normality has been in fact often conceived as construction that founds him on the adherence or less to the statistic norm: a mere abstract line, that sets a limit above what is normal is found; an example can be the diagnostic and statistic manual of the mental disorders. A criticism can be advanced to this concept, since it seems a certain dichotomic attitude is applied: our concepts of normality and abnormality, together with the labels and categories, are rooted on social constructions, elaborate on abstract concepts during some specific years from members of the society [32,33], and this can show "an ideological component" [5] for example to recognize that aspects of personality that were "the site where the seeds future of individual and social problems were sown and germinated" [6] and, accordingly, established as an object of intervention [1]. It's necessary to point out that people can introduce considerable statistic deviations from the statistic norm without being abnormal and/or pathological or unhealthy [34]. As we can see the themes of health and normality 


\section{Psychology \& Psychological Research International Journal}

are historically intertwined, and a static vision of health is centered on a concept of normality that individualize an inferior and superior thresholds of variability within which some indicators must be attested as normal or not. This concept is of nineteenth-century derivation and has been conditioned in the last decades from the biomedical approach that proposes the evaluation of the state of Health founded on the absence/presence of pathology. As a matter of fact the indexes of mortality in a population, the data on the invalidity and on the use of the services of care, have contributed in past to define the semantic field of the concept of health from a connected point of view to the condition of illness. From these perspectives the state of health has been considered as a baseline from which to depart for measuring degrees of discrepancy, but beginning from the fifties of the last century, the World Health Organization, (OMS) going besides the concept of "absence of illness", and the confusion between normality and health, proposed a definition of health "in positive", and it proclaims its identity it this way: it is not an absence of illness, but it is a state of physical, psychic, social and environmental wellness [35]. Such definition, underlining more dimensions, focused the complexity of this concept and has represented a point of turn.

\section{Ambiguity and Efforts to Draw a Distinction}

Some efforts to draw a distinction between these two notions have been made in a variety of contexts so the relationship among these two notions can be clarified, pointing out the points of contact and, then, specifying the ways in which they differ and the new point of view about these topic. Canguilhem [36] deepened both the ambiguity of the term health and the ambiguity of the term normality. The term health primarily has two different senses: it can be considered in absolute, as a normative concept that defines an ideal type of structure and organic behavior; this point of view remembers us the meaning of platonic derivation, and shows a point of contact with the concept of normality. The second meaning is the qualified health (alias "good" or "bad" health). In this case it deals with a descriptive concept that defines a certain disposition and the reactions of an individual organism towards possible illnesses. To the actual state, the word health can be considered ambiguous because it represents different and tangled concepts. How can this ambiguity be resolved? The word health today isn't conceptualized as absence of illness and is not conceptualized anymore even as is it something separate from the illness, but more often as expression of an integrative dynamics of illness and health, that we will go to explore. Perhaps in this meaning the health recalls more implicitly to a trial of continuous interaction between the person and the context, understood in its manifold aspects (cultural, social, report her, physical). The word health abandons then its ambiguity if it assumes the unique and representative characteristic of a trial that qualifies the "healthy operation". Canguilhem illuminates the overcoming position of the concept of health like an expression of the dynamicity of life, because he underlines how this concept passes from a focus on the contents and classifications to a focus on the activities: for the author the health is an "active presence", a try to evolve by promoting change, accepting the challenges of life and growing. Anderson [37] proposes some further specifications observing an idea of health that focuses on the dynamic continuous change, learning and development in the life cycle, also through phases of creation and destruction. This concept reveals to be the most modern and more akin to that specified by Canguilhem. Other attempts to go over the medical conception of health have been made by Ryff and Singer $[38,39]$; for these authors the health is not a medical matter, but rather a philosophical matter, and also these authors affirm that the human health is better understood as a dynamic and multidimensional dimension, not as a discreet static condition. Also the concept of normality has been considered ambiguous [36] because on one side it refers to statistic data, but from the other side it implicitly suggests the idea of value to pursue. Canguilhem speaks of the equivocalness of this word declaring that the meaning of normal, in the most usual sense of the term, is what meets the greatest part of the cases (of a measurable parameter/factor), but this term is equivocal since it designates, at once, a fact and a value attributed to this fact: a common factor assumes the value of an ideal; in addition in the biomedical field, the word "normal", over that the neutral statistic meaning, clearly communicates also the concept of healthy, and the health in this optics is not a quantitative matter, in excess or in defect, in comparison to the condition of illness. The health is statistically identified with a range of normal values and a subject is therefore considered healthy if it reenters inside this range. This intricate point of view obviously generates confusion. Antonovsky [40] also offers an interesting metaphor about health, comparing the situation of the sick to swimmers that grope in a river, while the residual category comes named as that of the healthy (presumably a great majority, at least in the western population) subjects. In this metaphor the preventive medicine is dedicated to those who are on the bank of the river but in danger of falling into the river, however we are all destined to die, to fall into the river, 


\section{Psychology \& Psychological Research International Journal}

and therefore Antonovsky said that a dichotomic classification is inappropriate, and created a different and particularly congenial theoretical base to clarify the concept of health. If, in reality, each of us is in the river and anybody on the shore, he is deduced that a dichotomic classification is unuseful, and proposed a model of a continuum: the health/sickness continuum, a more accurate conception according to this author; a conception that opens the street to a new theory [40].

Going beyond the dichotomy and the statistical norm, we can therefore refer to the thought of Canguilhem [36] to affirm that today what characterizes the health is the possibility to change and evolve, it is the tolerance towards the "infidelity" of the environment. Changuilhem say that the environment is "unfaithful" because life is not monotonous, predictable and a straight movement completely controllable: life ignores geometric rigidity, but it is the discussion and interaction with the environment (in which there are resources, but also unexpected obstacles and oppositions). Health consists in developing the margin of tolerance with respect to the environment's "infidelity". The more a person is "healthy", the more he/she is dynamic, in transformation, ready to the new, tolerant [13]. The Healthy persons are available for external stimuli, conceptualized not as a harmful element from which to protect themselves [13].

\section{New theories}

After having analyzed the ambiguities, the contact points, and the distinction between the concepts of health and normality, it becomes possible to perform a second discussion dedicated to the new theories about these two concepts. Today Bertini affirms that in virtue of the new awareness about the identity of the health is necessarily to create a more complex point of view, a new perspective that today the science points out us as a bivariate model. According to this vision the model of health is not in alternative to the model of illness: making reference to the historical excursus we can say that the presence of "Igea cannot remove the presence of Panacea" [20]. According to Bertini from a theoretical point of view it is necessary to overcome the line of continuity between health and disease, and focus on the fact that qualitatively there is the subsistence of two different dimensions that are in co presence; this concept can also be found in thought of various epistemologists as Canguilhem, who noticed that there are healthy biological norms and pathological norms, and that the latter are not of the same quality as the first. The conception of the quantitative continuum of health and disease is therefore no longer suitable today. As a matter of fact Canguilhem refused with numerous examples the thesis according to which there is a substantial continuity between health and pathology [36]. Also Gadamer observed that the illness cannot be defined by the standard values, gotten with the calculation of a mathematical average, that point out us what it is healthy: the continuum model is not adequate [14]. As a matter of fact today many scientists believe that the health and illness are not distributed along a bipolar continuum but are qualitatively different entities that can be co-present. The co presence can be expressed on a bivariate continuum [21-27] that must not be confused with the traditional bipolar continuum; Reiss and Gable [27] for example put in evidence that happiness and distress are better to understood as independent dimensions and not as opposite dimensions in a continuum. This deposes for the confirmation of a bivariate model. Mental health and mental illness are not situated to the opposite poles of an only continuum. The passage to a new vision of the Health open spaces for the elaboration of new ideas both on the plan of the theoretical understanding of the phenomena, both for the concrete application in the plan of the practice. The demonstration of the relative independence of the two dimensions puts to fire the central theme of the copresence of Health and illness and their possible integration. The bivariate model [21-27] invites us to study in a new way the relationship between positive affectivity and negative affectivity and invites us to reflect on the limits of the classical dichotomic vision. This model also introduces some new difficulties, like conceptual difficulties in considering the co presence of opposite, recognize and to accept that suffering does is a part of our life. With this new model is also possible to let emerge a more articulated frame of understanding of the relationship among the two dimensions however [31]. This passage also deals with understanding that while so much is worked on the epidemiological criterion, few has been done for individualizing and also to measure the dimensions and the dynamics of the health. In the psychological research area little attention has been turned on the characteristics of the health and the bivariate model [21-27] constitutes an occasion to face new horizons. Today the health can also assume the characteristics of "an active presence", of a reality whose identity doesn't depend on the static maintenance of norms to defend, but from the ability of change and development in the change: the dynamic evaluation of health as active presence, pointed out by Canguilhem. In synthesis we can think that in the thought of various Authors the presuppositions are created for overcoming the reductionism of the dichotomy that separates the 


\section{Psychology \& Psychological Research International Journal}

normal one from the deviant to meet a more complex point of view [20] with the evaluation of the health as a dynamic development and not as a static condition: a point of view in which health and suffering are placed in a new integrative vision. The ability to evolve is also a fundamental characteristic of health and as a matter of fact the human being much more it is "healthy", how much more changes and evolves [13]. Also about the specific concept of Normality there is little accord on its definition, because this concept results too tied to the culture and models of behavior, that don't keep in mind the flexible interactions between personality and circumstances [41], so Canguilhemit proposes then the new concept of normativity [36]. As we pointed out, this Author considers the health as a positive and vital dimension, linked to the ability to change, to innovate his/her own norms in the relationship with the environment, so he proposes to overcome the statistic concept of normality consolidating the dynamic concept of normativity: under conditions of "good health" there is the human power to produce constantly new norms. From this point of view the health is not normality, but is normativity: the availability to adapt with success in varied environments and to be able to transform them; the illness constitutes an impoverishment of such creativeness [42]. The concept of normativity points out the competence to found new norms; the author doesn't mean a reactive adaptation to the environmental eventualities, but a proactive tension. The subject doesn't feel healthy if he/she feels normal, but if she/he feel normative: able to follow new norms of life, and to evolve [36]. "Normativity" goes beyond the classical normal/deviant dichotomy and focus on the competence to change in front of the events of the life, in a process of personal growth. In synthesis this new perspective inspired to illustrate the vital dynamics of the subjects in the relationship with the environment and offers new points of view on the concepts of health and normality.

\section{Some Final Considerations}

The notions of health and normality have been historically examined and this excursus can be useful to delineate a summary that can support an interdisciplinary discussions. It is desirable a change of perspective that not only involves the field of psychology, but also other scientific areas where other health professionals work; the new dynamic evaluation of health, struggle against a dichotomic perspective [31] and deals with an open minded vision that gives prominence and interest to an ampler development of the subject, contemplating the possibility that temporary crisis can exist, but they are proper of the evolutionary phase of the subject. It deals with a vision in line with the paradigm of the complexity, in opposition to the biomedical approach: a model that doesn't help to understand the complexity of the human being [43]. We can also underline that the static concept of normality is based on shared labels of an epoch, created to define what it is normal or not, labels rooted on social constructions: abstract concepts elaborated during the years from members of the society [33] notions that are socially transformed with time, to soon leave their place to others conceptions; this is evident for the different diagnostic categories created for the Diagnostic Statistic Manual of the Mental Disorders (for example those concerning sexual preferences). Fortunately, today there is growing awareness of this aspects, and as a matter of fact also in the latest version of the Diagnostic Statistical Manual of Mental Disorders (DSM V) it is stated that diseases (and even normality) are defined in relation to the cultural context because the culture of belonging provides interpretative contexts that shape the experience and the manifestation of the symptoms and behaviors that constitute the criteria for the diagnosis [44]. According to the DSM V the diagnostic evaluation (of normality or abnormality) must therefore take more into account the fact that the boundaries between normality and pathology are different from a culture to another for specific types of behavior. It has also been pointed out that the decision regarding the attribution of abnormalities of behavior and the need for clinical attention depends on cultural norms internalized by the individual and applied also by others (even family members and friends), just because there is more attention to the dynamics according to which the culture can influence acceptance or refusal of a diagnosis and a treatment, affecting in this way also the course of the disease and consequently the processes of healing [44]. Thus, emergesa more attentive point of view to the complexity of the human being that evolves in the cultural context, also specifying considerations regarding the potential differences between men and women in the expression of mental illness [44]. Precisely in light of these considerations the new dynamic model of normativity helps us to better understand the human ability to evolve: how much more a person is healthy so much more he/she is able to change and find appropriate solutions to develop in his/her context of life.

\section{References}

1. Banicki K (2017) The character-personality distinction: An historical, conceptual, and functional investigation, Theory \& Psychology 27(1): 50-68. 


\section{Psychology \& Psychological Research International Journal}

2. Hafen M (2016) Of what use (or harm) is a positive health definition? J Public Health 24: 437-441. DOI 10.1007/s10389-016-0741-8

3. Batstra L, Frances A (2012) Holding the line against diagnostic inflation in psychiatry. Psychotherapy and Psychosomatics 81: 5-10.

4. Genuis S K (2016) Bronstein J., Looking for "normal": Sense making in the context of health disruption, Journal of the Association of information science and tecnology 68(3): 750-761.

5. Danziger K (1990) Constructing the subject: The historical origins of psychological research. Cambridge, UK: Cambridge University Press.

6. Danziger K (1997) Naming the mind: How psychology found its language. Cambridge, UK: Cambridge University Press.

7. Engel GL (1977) The Need for a New Medical Model: a challenge for biomedicine Science 196: 129-136.

8. Engel GL (1980) The clinical application of the biopsychosocial model, American Journal of Psychiatry 137: 535-544.

9. Engel GL (1992) How much longer must medicine's science be bound by a seventeenth century world view? Psychoterary and Psychosomatics 57: 316.

10. Lipowsky ZJ (1977) Psychosomatic medicine in the seventies. In american journal of Psychology, 134233.

11. Solano L (2001) Tra mente e corpo, Raffaello Cortina, Milano.

12. Searle JR (1998) Mind language, and society: Philosophy in the real world, Basic Books, Boston.

13. Florita M (2011) L'intreccio. Neuroscienze, clinica e teoria dei sistemi dinamici complessi, Franco Angeli, Milano.

14. Gadamer HG (1993) Dove si nasconde la Salute. Tr. it. Raffaello Cortina, Milano 1994.

15. Erikson ER (1963) Childhood and society (2nd ed.) New York: Norton.

16. Spinosi N (2005) Mentalità della sopravvivenza in psicoterapia, Firenze University Press.
17. Vincenti E (2013) Il gruppo come proprietà emergente, Ricerca Psicoanalitica, XXIV,1/2013: 1226

18. Good BJ (1999) Narrare la malattia: lo sguardo antropologico sul rapporto medico paziente. Torino: edizioni di Comunità.

19. Saita E (2011) Pensare alla Salute e alla Malattia, legami tra mente, corpo e contesto di appartenenza, Educatt.

20. Bertini M (2012) Psicologia della Salute, Raffaello Corina Editore, Milano.

21. Davidson JR (1992) Anterior cerebral asymmetry and the nature of emotion Brain e cognition 20(1): 125 151.

22. Davidson JR (1994) On emotion, mood, and related affective constructs, in The nature of emotion: Fundamental questions. Series in affective science. Ekman P, Davidson R, New York, NY, US: Oxford University Press, 51-55.

23. Gray JA (1994) Three fundamental emotion systems, in The nature of emotion: Fundamental questions. Series in affective science. Ekman P, Davidson R, New York, NY, US: Oxford University Press, 243-247.

24. Cacioppo JT, Berntson GG (1994) Relationship between attitudes and evaluative space: A critical review, with emphasis on the separability of positive and negative substrates. Psychological Bulletin 115(3): 401-423.

25. Hoebel BG, Bartley G, Rada PV, Mark GP, Pothos EN (1999) Neural systems for reinforcement and inhibition of behavior: Relevance to eating, addiction, and depression. In Kahneman D.; Diener E.; Schwarz, Norbert, Well-being: The foundations of hedonic psychology, New York, NY, US: Russell Sage Foundation, pp. 558-572.

26. Ito TA, Cacioppo JT (1999) The psychology of utility appraisal, in Kahneman, D.; Diener, E.; Schwarz, Norbert, Well-being: The foundations of hedonic psychology, (pp. 558-572). New York, NY, US: Russell Sage Foundation, 471-488.

27. Reiss HT, Gable SL (2003) Toward a positive psychology of relationships, in Keyes CL, Haidt J, Flourishing. Positive Psychology and the Life WellLive, APA, Washington, DC. 


\section{Psychology \& Psychological Research International Journal}

28. Vachá J (1978) Biology and the Problem of Normality, Scientia 72: 823-846.

29. Wright BA, Lopez SJ (2002) Widening the diagnostic focus, in Snyder CR, Lopez SJ Handbook of positive Psychology, Oxford University Press, New York , 2644.

30. Freud A (1936) l'Io e i meccanismi di difesa in Opere, Torino, Bollati Boringhieri 1.

31. Irtelli F (2016) Illuminarsi di Ben-essere, Armando Editore. Roma.

32. Freides D (1960) Toward the elimination of the concept of normality. Journal of Consulting Psychology 24(2): 128-133.

33. Widiger TA, Trull TJ (1991) Diagnosis and Clinical assessment in «Annual Review of Psychology 42: 109134.

34. Maddux JE (2002) Stopping the madness: Positive psychology and deconstruction of the illness ideology and DSM .In Snyder CRLopez, SJ Handbook of Positive Psychology. Oxford University Press, New York, pp1323.

35. WHO, Word Health Organization (1948) Costitution of the World Health organization, New york.

36. Canguilhem G (1966) Il normale e il patologico. Tr.it. Einaudi, Torino1998.
37. Anderson R (1984) Health promotion: an overview. European Monographs. In Health Education Research 6(4): 4-119.

38. Ryff CD (1989) Happiness is everything, or is it? Explorations on the meaning of psychological wellbeing In Journal of Personality and Social Psychology 57(6): 1069-1081.

39. Ryff CD, Singer B (1998) The contours of positive human health In Psychological Inquiry 9(1): 1-28.

40. Antonovsky A (1996) The salutogenic model as a theory to guide health promotion. In Health promotion International. Oxford University Press 11(1): 1118.

41. Gollin S, Stahl G, Morgan E (1989) On the Uses of the Concept of Normality in Developmental Biology and Psychology, Advances in child development and behavior 21: 49-71.

42. Porro M (1998) Canguilhem: la norma e l'errore, Introduzione, in Caugilhem G., Il normale e il patologico, Einaudi, Torino.

43. Minolli M (2015) Essere e Divenire, Franco Angeli.

44. American Psychiatric Association (2013) Diagnostic and Statistical Manual of Mental Disorders, DSM-5. American Psychiatric Association Publishing, Washington, DC.

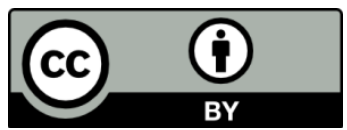

